\title{
Total darkness as an aversive stimulus condition for the squirrel monkey
}

CHRISTOPHER E, PARKER

SAN DIEGO STATE COLLEGE

Squirrel monkeys were maintained in a dark chamber for from four to 39 continuous days. When light was used as a reinforcer, a high rate of lever pressing, inversely proportional to the duration of light per response, was obtained. An extreme reluctance to consume food or water in total darkness was observed. The complete absence of light is apparently an aversive stimulus condition for this species.

It has been well substantiated that for the rat an increase in the ambient light level can act as a reinforcer for operant responding. The reinforcing and other effects of changes in illumination on the behavior of rodents has been investigated at some length, and recently reviewed by Lochard (1963). Fewer studies are available, however, indicating that an increase in illumination will reinforce operant responding by other species. The rhesus monkey, at least, will perform an operant response which increases the ambient light from some lower level to a higher level (Moon \& Lodahl, 1956). An attempt to replicate the phenomenon in the squirrel monkey (Scimiri sciureus) turned up some unexpected results which led to a series of experiments on the aversiveness of total darkness for the squirrel monkey.

\section{Synopsis of prior studies}

The initial studies employed a procedure similar to that which Moon \& Lodahl (1956) employed with rhesus monkeys. The $\mathrm{S}$ was placed in a lightproof, air conditioned box measuring 24 in. $x 24$ in. $x 18$ in. with food, water, and milk available at all times. Two 7-1/2 w lamps behind frosted glass panels served as light sources inside the chamber. One lamp, the ambient source, was controlled from outside the box, the other, reinforcement source, by either of the two identical levers mounted next to each other on the inside of one wall of the chamber. One lever was designated as active and turned on the reinforcement lamp for a metered interval of time regardless of how long the lever was held down. The other, dummy, lever did not increase the illumination. The relative position of the active and dummy levers was reversed daily.

Various Ss were kept in the apparatus from four to as many as 39 continuous days. The series of four, one-S studies yielded the following data: (1) The lever which turned on an additional light source within the box, thereby increasing the illumination level, was pressed significantly $(p<.01)$ more times than a similar lever which did not increase the illumination level. (2) When the ambient light source was turned off, making the chamber completely dark between reinforcements, response rate increased significantly $(p<.01)$. (3) When the duration of light per lever press was manipulated, response frequency was inversely proportional to the duration of light per response over the range $1 / 2 \mathrm{sec}$. to $1 \mathrm{~min}$. (4) At the shorter durations of light per response, response rate was maintained at a high level. For example, at a duration of $5 \mathrm{sec}$. per response, the mean rate for $24 \mathrm{hr}$. was $375 \mathrm{re-}$ sponses per hour. Thus, the reinforcement light was kept on for possibly 13 of the $24 \mathrm{hr}$. The S's behavior was highly similar to that reported by Sidman (1953) for rats postponing a shock in a non-signaled avoidance situation. (5) Food, water, and milk consumption was extremely low on days when the light duration was short (5 sec. or less). Some experiments had to be terminated after the $\mathrm{S}$ went for $48 \mathrm{hr}$. without consuming more food-mash, milk, and water than could be accounted for by evaporation. In fact, when the response lever no longer yielded an interval of light, i.e., during extinction, no food at all was consumed. (6) Vocalizations frequently occurred immediately after light termination, and bursts of responses frequently occurred immediately after noises in the laboratory.

The apparatus was located in a dark-room and a view-point in the box allowed observation of the S's behavior while the chamber was illuminated. At the shorter durations of light per response Ss were observed to turn on the light, pick up a piece of food and begin to eat. When the light went off observation was not possible, of course, but when the light came on again the $\mathrm{S}$ invariably did not have the food in hand. By the time the food was relocated and picked up again, the light would go off and the cycle was repeated. Thus, the seriously curtailed food consumption was apparently due to a reluctance to eat in the dark. It was hypothesized that when faced with the alternative of obtaining food and water in the dark or remaining in the light without food and water, squirrel monkeys would prefer the latter. It was this possibility which was investigated in the experiment to be reported below. Method

The apparatus described above was modifed in order to more directly pit food against light. The levers were removed and a horizontal perch inserted near each end of the box a few inches from the floor. A switch connected to the perch farthest from the food-cup kept the light on continuously as long as the S's weight was on that perch. The chamber was in complete darkness at all other times. A clock recorded the total amount of time $S$ spent on the light perch, i.e., the one which 
controlled the light. The food and water dispensers at the other end of the box could not be reached from the light perch, however, they were in full view. The food, Purina monkey chow, was made into a loose mash or gruel in order to prevent its being carried away from the food-cup to be consumed. In this situation, then, the $S$ could obtain food and water or light, however, not both simultaneously.

For the first three continuous days the ambient light was on for $24 \mathrm{hr}$. per day to allow adaptation to the situation. It is assumed that this was sufficient time for $S$ to become thoroughly familiar with the inside of the small chamber. During these three days, the animal ate well and spent only 9,3 , and 1 percent of each $24 \mathrm{hr}$. period on the perch which would later result in illuminating the chamber. Ss were observed during this time to be extremely inactive, spending long periods sitting on the perch next to the food-cup and water nipple. On the fourth day, when the light was made contingent upon being on the light perch, the light was on almost the full $24 \mathrm{hr}$. No water was consumed, however, some food was taken from the food-cup. Most of the missing food was on the floor in front of the food-cup. S was apparently dipping the hand into the gruel, in the dark, for it was observed licking the gruel off the hand while sitting on the light perch. Since very little food was actually consumed on each trip to the food dish, the S could be observed feeding in this manner at practically any time of the day. These data from a naive male were replicated in all respects with a female who had been in the apparatus during one of the lever pressing studies. In all, five different squirrel monkeys were used in the series of experiments. All Ss were reared in the light and believed to have normal pattern vision as attested to by successful object-quality discrimination learning either before or after serving as Ss in this experiment. None of the Ss had served in sensory deprivation experiments prior to this experiment.

\section{Discussion}

The higher response rates in complete darkness suggest that the onset of illumination in a completely dark environment is much more reinforcing than is an increase in illumination in a semidark environment. At least part of the reinforcing effect of light onset in this situation may be attributed to its being instrumental to, or necessary for, the consumption of food and water. Although not "tested to death" it seems likely that the squirrel monkey might remain in the light and literally starve to death rather than endure total darkness. This extreme reluctance to eat in the dark may be specific to the squirrel monkey as macaques (Ganz \& Riesen, 1962) and chimpanzees (Riesen et al, 1951) have been successfully reared in complete darkness without any apparent eating difficulties. Perhaps also, the squirrel monkey would find no difficulty eating in the dark if dark-reared from infancy.

In a recent article on punishment, Soloman (1964) observed that few punishments other than electric shock have been used, and called for the study of the effects of other kinds of punishments. For the squirrel monkey, and other visual species, complete darkness might be an effective form of punishment. Physiological studies most likely would show that darkness would result in increased heart rates, respiration rate, and other signs of activation or arousal, perhaps indicating that darkness is a fear-arousing stimulus condition for the squirrel monkey.

Taken together, the series of experiments seem to indicate that for the squirrel monkey, darkness is an aversive stimulus condition. The aversiveness is, perhaps, further attested to by the violent resistance met in attempts to put the monkeys back into the chamber once they had served as Ss.

\section{References}

Ganz, L., \& Riesen, A. H. Stimulus generalization to hue in the dark-reared macaque. J. comp. physiol. Psychol., 1962, 55, 92-99.

Lochard, R. B. Some effects of light upon the behavior of rodents. Psychol. Bull., 1963, 60, 509-529.

Moon, L. E., \& I.odahl, T. M. The reinforcing effect of changes in illumination on lever pressing in the monkey. Amer. J. Psychol. $1956,69,288-290$.

Riesen, A. H., Chow, K. L., Semmes, J., \& Nissen, H. W. Chimpanzee vision after four conditions of light deprivation. Amer. Psychologist, 1951, 6, 282. (Abstract)

Sidman, M. Avoidance conditioning with brief shock and no exteroceptive warning signal. Science, 1953, 118, 157-158.

Soloman, R. L. Punishment. Amer. Psychologist, 1964, 19, 239253. 